\title{
Experiencias de vida de consumidores de drogas portadores de VIH*
}

\author{
Kornblit, A.L. \\ Instituto de Investigaciones Gino Germani. Facultad de Ciencias Sociales. Universidad de Buenos Aires. \\ Docente investigadora. \\ * Trabajo realizado con la financiación de la Secretaría de Ciencia y Técnica. Subsidio BID 802-OC/AR-PICT 00021. \\ Enviar correspondencia a: \\ Ana Lía Kornblit. Moldes 1243. (1426) Buenos Aires. Argentina. T.E.: 54114783 0344; e-mail: kornblit@mail.retina.ar
}

\begin{abstract}
Resumen
El objetivo del trabajo es reconstruir las trayectorias vitales de una muestra de personas que viven con el VIH, cuya vía de infección fue el consumo de drogas por vía endovenosa, con el fin de relevar los significados atribuidos por ellas a aspectos clave de dichas trayectorias. Se tomaron quince entrevistas en profundidad, en las que las personas relataron sus experiencias de vida. Los relatos fueron analizados siguiendo los lineamientos de la Grounded Theory. La familia y el grupo de consumo surgen en el análisis como una dimensión clave con respecto a los significados atribuidos al consumo y a sus consecuencias, que se enlaza con la asunción del cambio a partir del diagnóstico de seropositividad. El análisis permitió construir una tipología acerca de dichos cambios, que incluyó: 1 . Cambios en el eje del hacer; 2. Cambios en la esfera afectiva; 3. La dificultad del cambio identitario; 4 . El no cambio.
\end{abstract}

Palabras clave: adicción endovenosa, sida, grupo familiar, grupo de consumo

\section{INTRODUCCIÓN}

E I objetivo de la investigación que dio pie a este trabajo fue explorar las experiencias de vida de personas afectadas por el sida, infectadas a través del consumo de drogas por vía endovenosa. Se buscó reconstruir sus trayectorias vitales y los significados atribuidos por ellas a aspectos clave de dichas trayectorias, en cuyo transcurso sucedieron, en un determinado momento, la infección por el VIH, el diagnóstico de seropositividad y el tratamiento para el sida.

\section{Abstract}

This paper is aimed at reconstructing different life experiences from a sample of HIV seropositive subjects that have been HIV-infected through intravenously administered substance dependence, so that, through reconstruction, a plotting of meanings subjects give to key aspects of their life experiences could be identified. Fifteen in-depth interviews have been carried out to gather subjects' life experiences. Thereafter, stories have been analyzed pursuant to the Grounded Theory guidelines.

Analysis suggests that both family group and substance consumer group are key dimensions with regard to meanings attributed to substance consumption and consequences thereof -a dimension intertwined with subjects' assumption of change caused by their having been diagnosed as seropositive carriers.

By means of analysis, a change-based typology could be constructed including: 1. Changes occurred within the sphere of subjects' activities. 2. Changes occurred within the sphere of subjects' affects. 3. Difficulties caused by the identity change. 4. No changes.

Key words: intravenous substance dependence - AIDS - family group - consumption group.

Los relatos de vida nos parecieron un instrumento adecuado para relevar dichos significados. La elección de esta metodología se vincula con el supuesto acerca de que la identidad es un esfuerzo organizado reflexivamente, que consiste en el "sostenimiento de narrativas biográficas coherentes pero continuamente revisadas" (Giddens, 1991). Si bien forman parte del enfoque biográfico (Sautú, 1999), estos "relatos" se definen como formas más concisas que, por ejemplo, las "historias de vida", dado que, si bien son respuesta a la demanda que se formula a los entrevistados de que describan sus trayectorias vitales, existe en ellos 
un hilo conductor implícito, dado por el objeto de estudio, en este caso: la experiencia subjetiva del sida.

Los significados relevados no son unívocos, ni se presentan a los sujetos como dados, sino que son construidos por ellos en el intento de fijar un sentido al flujo de los acontecimientos de sus vidas, entre la multiplicidad de sentidos posibles. En esta construcción intervienen elementos extraídos de la historia individual, de las experiencias compartidas y de los sistemas de creencias y valores de los grupos que los circundan. Reconstruir, en la medida en que "la opacidad intrínseca a las relaciones sociales" (Laclau, 1991) lo permita, los supuestos y los mundos posibles en los que los relatos se producen, es el objetivo de los análisis que siguen. Esta tarea será siempre parcial y mediatizada por la propia implicación de los investigadores en los relatos, dado que formamos parte de la realidad social que pretendemos estudiar.

La elección de la metodología cualitativa, por otra parte, se vincula con el supuesto de que en el mundo posmoderno, siguiendo a Foucault (1990), coexisten en un mismo espacio un gran número de mundos posibles fragmentados o que se yuxtaponen o superponen entre sí. Esa "heterotopía" mal puede ser reconstruida a partir de una meta-narrativa, como dice Lyotard (1985), que aspire a dar cuenta de totalidades.

Los relatos de vida que se analizan a continuación fueron hechos por personas cuya vía de infección del VIH fue el consumo de drogas por vía endovenosa. Todas ellas están o han estado en tratamiento por alguna/s enfermedad/es marcadora/s del sida. El plazo transcurrido a partir del diagnóstico de seropositividad varía desde uno a trece años. Se tomaron quince entrevistas en profundidad, cuya duración osciló entre dos y tres horas. El material fue grabado y desgrabado posteriormente para su análisis, realizado siguiendo los lineamientos de la Grounded Theory (Glaser y Strauss, 1967).

Los entrevistados fueron reclutados de maneras diversas: a través de conocidos, utilizando la técnica de bola de nieve dentro de servicios hospitalarios de atención de sida. Su extracción social es en la mayor parte de clase baja. Seis de los diez tienen estudios secundarios incompletos y uno no terminó la escuela primaria. Los tres restantes finalizaron la escuela secundaria. Sus edades van desde los 20 a los 33 años. Son todos varones debido a la imposibilidad que tuvimos de reclutar mujeres que afirmaran haber sido contagiadas por esta vía.

\section{LA FAMILIA DE ORIGEN}

Un aspecto clave de estos relatos es el énfasis que sus autores ponen en una conflictiva relación con su familia de origen y una progresiva entrada en el "mundo de la droga", y en algunos casos del delito, como búsqueda de una socialización secundaria que ofreciera alternativas que no vislumbraban a partir de su socialización primaria.

Para entrar en la comprensión de ese fenómeno ayuda el hacer un repaso de los cambios que afectan a la familia actual. En Sociologie de la famille contemporaine De Singly (1993) caracteriza a las familias actuales del mismo modo como lo había hecho Durkheim (1888) un siglo atrás, por un doble movimiento: la privatización, dada por una mayor atención a la calidad de las relaciones intrafamiliares, y a la vez una mayor intervención del Estado, en su carácter de controlador del funcionamiento familiar (Donzelot, 1977). La familia es cada vez más un ámbito en el que los sujetos aspiran a proteger su individualidad, a la vez que les preocupa la gestión de sus territorios personales al interior de la vida doméstica, vale decir, se esfuerzan por delimitar un ambiente que sea protector con respecto al afuera y al mismo tiempo no invasivo en cuando a los espacios de cada uno.

Si para los seguidores de Durkheim la característica de la familia actual es el énfasis en lo relacional, la tesis de Ariès, (1960) publicada en su libro: L'enfant et la vie familiale sous I'Ancien Régime es, por el contrario, que es la escuela la que instaura una nueva relación con el niño y con la familia. La familia actual sería incomprensible, para este autor, fuera de la historia de la escolaridad. El "sentimiento de familia" y el "sentimiento de la infancia", surgidos de la ideología escolar, dan forma a una mayor preocupación por lo interpersonal y por gestionar las condiciones para una buena socialización de los niños. El cambio de la familia 'antigua' a la 'moderna' se opera por mediación de un cambio de relación con el niño. La familia se transforma profundamente en la medida en que modifica sus relaciones internas con el niño. Hay que tener en cuenta también que la escolaridad extendida actual promueve la cohabitación de las generaciones.

Las estrategias educacionales, de las que las estrategias escolares forman parte, se sitúan en el centro de las estrategias de reproducción social. Según Bourdieu (1980) se da un "modo de reproducción familiar a componente escolar", que privilegia el rol determinante del "capital escolar" en la fijación del valor de los individuos.

Ahora bien, reconstruyendo los grupos familiares de los entrevistados, ninguna de estas condiciones se cumple: no existe en ellos ni una preocupación por el desarrollo de las individualidades en un ambiente protegido, ni un énfasis en las estrategias escolares, presentes especialmente en las familias de clase media. Existe un consenso social que hace que en los relatos surja una fuerte crítica al hecho de que no se cumpla con estas reglas, como si el modo de funcionamiento de las familias de clase media, por lo menos en estos aspectos, fuera el modelo vigente para todas, aun las 
de clase baja. Sin embargo, estas características familiares están ausentes en las familias de los entrevistados.

Ello plantea la pregunta de cómo son, según el relato de los entrevistados, sus grupos familiares. En todos los casos, y sin que esto implique ubicar a la familia de origen como agente causal en la "carrera" del drogadicto, existe una caracterización del núcleo familiar como carencia afectiva. Estamos planteando aquí la incidencia de la imagen de los entrevistados acerca de su familia de origen como un grupo social en el que "faltaba algo", lo que influye sobre la búsqueda, a través del consumo de drogas, de un algo indefinido que llene ese vacío.

Llegar a sentirse uno mismo y al mismo tiempo pertenecer a un grupo es la doble dimensión que subyace al proceso de constitución de la identidad. Plantear la drogadicción como una práctica relacionada con la búsqueda de esa doble dimensión equivale a relacionarla, aunque no en una causalidad mecánica, con dicho proceso.

Con independencia de las características "reales" de desprotección que pueden haber estado vigentes en los núcleos familiares de los entrevistados, asumimos aquí el punto de vista de que la práctica del consumo implica la creencia en la posibilidad de lograr a través de ella una posible protección imaginaria frente a las sensaciones dolorosas de fracasos psico-socioafectivos (Delor, 1997).

Las familias de los entrevistados son descritas en todos los casos como conflictivas. Dicho conflicto se asocia a distintos aspectos: muertes, separaciones (que llevan en algunos casos a la separación también de los hermanos), familias ensambladas mal integradas, violencia física y verbal, alcoholismo, etc. Son todos aspectos que redundan en la sensación de soledad, abandono y falta de continencia afectiva vivida por los entrevistados.

La imagen conflictiva del padre coincide con la representación social acerca de los padres (varones) (cf. Kornblit et al., 1998). A pesar de que se rechaza la imagen autoritaria del pater familia, se los acusa en general de debilidad e impotencia, y de no estar a la altura de la "gran tarea paternal", caracterizada por una especial exigencia en la que deberían a la vez seguir constituyendo la "columna vertebral de la familia" y ser afectivos con los hijos pequeños y contenedores con los hijos adolescentes.

La representación social de las madres se ha mantenido a lo largo del tiempo con menos modificaciones. La función materna se piensa como asumida por alguien que ha deseado al niño. El deber referido a su rol sigue estando vinculado con el afecto incondicional a los hijos, independientemente de su edad. Entre los entrevistados, ninguna de las madres, sin embargo, cumple con esta expectativa. Esta no asunción por parte de las madres de los entrevistados de su rol es fuertemente reprochada por ellos, apoyándose en algunos casos en la comparación con otras personas que cumplieron con dicha expectativa de amor sin límites, por ejemplo, en algunos casos, las abuelas paternas.

En todos los relatos surge la imagen de la familia de origen como ente carente de protección que da lugar a una búsqueda que se establece, a partir de esta vivencia, de espacios contenedores. En casi todos ellos se menciona una persona recordada como opuesta a esta falta afectiva. En algunos casos se trata de una abuela, en otros de un padre que se recupera después de una ausencia, pero estas experiencias afectivas no alcanzan para paliar la desazón por la falta percibida. En algunos de los entrevistados este espacio es llenado - si bien limitadamente en el tiempo - por una pareja.

El análisis tanto de los grupos familiares de origen como de los constituidos por los entrevistados verifica que "el plural se impone hoy en día cuando se habla de la familia" (Segalen, 1996). No hay un modelo sino varios. Familias que sostienen la conyugalidad a pesar de fuertes conflictos; familias monoparentales (con la madre como pivote, sostenida por su red de parentesco, o con el padre a cargo del hijo por viudez); familias ensambladas... formas distintas en las que, aun en su diversidad - por lo menos en la visión de los entrevistados -, prevalece la falta de cohesión y de solidaridad, lo que provoca la nostalgia por el ideal de la familia tradicional. Según Giddens (1999), esta nostalgia parte de una idealización del pasado, ya que las familias rotas eran tan comunes en el mundo occidental en el siglo XIX como ahora, aunque el motivo principal fuera la muerte y no tanto las separaciones.

Para el mismo autor el tema de la familia ha sido una arena para la controversia de las posturas políticas. El punto de vista de la derecha es que la destrucción de la familia tradicional provoca la crisis social. El punto de vista de la socialdemocracia, en cambio, es aceptar las formas familiares diferentes que han ido surgiendo a partir de la segunda mitad del siglo XX, considerando que se trata de un polimorfismo que en sí mismo no es ni bueno ni malo. Lo que sí puede plantearse es que "fragiliza" las trayectorias de vida de personas como los entrevistados y eventualmente los acerca a "zonas de riesgo", incluyendo distintas formas de delito. Puede decirse que es la falta de continencia que los sujetos asocian con las estructuras familiares no tradicionales, sin que tal falta esté forzosamente vinculada a dichas estructuras. 


\section{EL GRUPO DE CONSUMO}

El grupo de consumo es descrito como lo que permite arribar a una sensación a la vez de libertad y de comunión con otros. La oposición entre la familia de origen y el grupo de consumidores es, en los primeros momentos de la adicción, equiparada a la oposición entre la insatisfacción y el placer. Es importante en la caracterización del polo del placer, el aspecto de aprendizaje en el consumo, vehiculizado en general por un personaje al que se caracteriza como líder por ser más grande, más experimentado, más arriesgado, etcétera. Este "héroe", citado por casi todos los entrevistados, alimenta su influencia sobre ellos en la inseguridad de los seguidores. Como dice Moscovici "cuanto más insegura se siente una persona en sus aptitudes sensoriales e intelectuales, más dispuesta está a aceptar la influencia de alguien a quien atribuye capacidades sensoriales e intelectuales superiores" (1996: 50). Estas capacidades superiores son descritas en todos los casos como el "ser canchero", "ser piola", "tener calle", en suma, cualidades admiradas a partir de la sensación de inexperiencia de jóvenes que salen de una socialización primaria que los marca con la incertidumbre de no saber si son queribles o son capaces (recordemos los frecuentes fracasos escolares que anteceden al inicio en el consumo). El mismo Moscovici afirma, y esto se aplica estrechamente al tema que nos ocupa: "El individuo que tiene un entorno a su favor (...) es capaz de resistir las presiones sociales y de escapar a la incomodidad resultante de su interacción con otros (...) La noción de incertidumbre desempeña en psicología social un papel análogo al de norma en antropología o al de escasez en economía (...) La certeza es un recurso difícil de obtener y para lograrla las personas se asocian o se someten a otros" (Moscovici 1996: 53).

La decepción frente a la falta de reconocimiento afectivo durante la socialización primaria puede pensarse como uno de los factores explicativos, que según los entrevistados, los empuja a la búsqueda de gratificaciones (afectivas y sensoriales) o de continencia en el grupo de pares, donde se inician en el consumo.

\section{LA VUELTA A LOS VALORES FAMILIARES}

Llama la atención que tras el impacto producido por el diagnóstico de seropositividad, vivido en el mismo momento o después como la consecuencia de haberse volcado a la subcultura juvenil, los entrevistados recuperan, en la mayor parte de los casos, el discurso de los valores familiares. Este planteo en lo ideal se acompaña a veces por un intento de acerca- miento a la familia de origen, tratando de recuperar vínculos que en algunos casos están muy debilitados.

Puede tomarse en cuenta para analizar este hecho la clasificación que realizan Hall y Jefferson (1983) acerca de los escenarios que influyen en las subculturas juveniles:

a) la cultura hegemónica, que implica la distribución del poder cultural en la sociedad en sentido amplio, en sus relaciones con las instituciones laborales, educativas, etcétera;

b) las culturas parentales, que incluyen las normas de conducta y los valores vigentes en el medio social de origen de los jóvenes;

c) las culturas generacionales, que se refieren a los valores y patrones de conducta que los jóvenes adquieren a través de sus experiencias, particularmente en sus espacios de ocio.

Los significados que se otorgan al ámbito laboral, a la escuela y a las instituciones sanitarias son algunas de las referencias a la cultura hegemónica que surgen en los relatos de los entrevistados. Estas referencias implican, en especial con respecto a los dos primeros ámbitos, atributos negativos, en la medida en que la mayoría expresa la sensación de haber sido expulsados de dichos espacios.

Hemos puntualizado también la imagen de la familia de origen como núcleo del que la mayoría de los entrevistados se queja, en la medida en que no ha satisfecho sus necesidades de afecto y pertenencia. La cultura generacional, transmitida en este caso por el grupo de consumo, que es en general el grupo del barrio, representan la alternativa que los jóvenes entrevistados encuentran frente a la sensación de dificultad de inserción en los espacios "oficiales" y de desamparo con respecto a la familia de origen. La vuelta a la cultura parental a partir del sida se produce tras la condena de la cultura generacional, en la medida en que han representado para ellos la amenaza de la muerte. Esta revalorización se acompaña en algunos casos del proyecto de un desempeño de roles, por ejemplo el de padres, que mejore el de sus progenitores.

La valorización de la familia se realiza sobre la base de la imagen de la "familia monolítica" (Langellier y Peterson, 1997), en la que se otorga prioridad al núcleo unido por lazos de amor, protegido en un hogar, y organizado por la división social del trabajo. Se proyecta así una norma invariable, homogénea, que no toma en cuenta las desigualdades de género ni los efectos de las condiciones socioeconómicas sobre los grupos domésticos. Hareven (1991) ha insistido en este sentido que esta imagen corresponde al modelo burgués de mediados del siglo XIX, desconociéndose otros modelos familiares distintos.

La primera regla esencial de la vida familiar según dicho modelo consiste en que frente a algún padeci- 
miento se puede contar con la familia. La falta de cumplimiento de esta expectativa es precisamente lo que reprochan casi todos los entrevistados a sus grupos familiares. La reivindicación de los valores implícitos en las culturas parentales hace que, ateniéndonos a los desarrollos de los estudios culturales, pueda hablarse de una subcultura de la droga y no de una contracultura en relación con dicho mundo. Las contraculturas, estudiadas por Moscovici (1996) como "minorías activas", construyen sus posiciones en oposición a la cultura hegemónica y las convierten en propuestas de cambio. Las subculturas transgresoras, en cambio, se sitúan dentro del marco de la visión mayoritaria, aun cuando sus sostenedores se vivan como excluidos del sistema. Los teóricos de la escuela de Birmingham, como por ejemplo Cohen (1972), planteaban que "la función latente de las subculturas juveniles es expresar y resolver, aunque sea mágicamente, las contradicciones que permanecen escondidas e irresueltas en el seno de la cultura parental, por ejemplo los elementos de cohesión social destruidos por ellas" (pág. 23).

Los relatos de los entrevistados muestran que, como dice Feixa (1999), el recurso de la subcultura de la droga no ofrece una resolución de tales contradicciones, sino la vuelta a lo mismo de lo que se partió, si bien en versiones que se intentan corregir. El potencial transformador de la subcultura de la droga frente a la cultura hegemónica se invalida, pues, a partir del recorrido de los entrevistados. La comunión lograda en el grupo, que es uno de los valores reivindicados, es ilusoria y efímera. No obstante, a pesar de esa reivindicación de los valores de las culturas parentales, en algunos casos el grupo de consumo es mencionado como el espacio social en el que aún en la actualidad es posible para los entrevistados experimentar el sentimiento de comunión y solidaridad vivido en las "buenas épocas".

Imaginarse el asombro, el temor y el dolor al ver diezmarse el grupo por obra del sida lleva a comprender mejor la ambivalencia entre el rechazo y la defensa del grupo. Rechazo por la identificación con un estilo de vida unido a lo ominoso y su defensa como sostenedor del apoyo social, no encontrado en la familia de origen.

\section{LOS CAMBIOS ASUMIDOS A PARTIR DEL DIAGNÓSTICO DE SEROPOSITIVIDAD}

Los relatos analizados pueden clasificarse según las propuestas de cambios que se formulan los entrevistados al enfrentarse con el diagnóstico de seropositividad, ya sea efectivamente realizados o como proyectos. Cada uno de estos tipos de cambio se vincula con una particular modalidad de gestión frente a la enfermedad y con un modo de apreciar la dimensión familia-grupo de consumo.

Como surge de los relatos, los cambios pueden categorizarse en cuatro tipos:

\section{Cambios en el eje del hacer}

Cambios que tienen por eje el hacer algo diferente en relación con las actividades llevadas a cabo en la fase anterior (la etapa del consumo). En estos relatos prevalece la idea de la identidad a través del hacer. Se llega a ser como se quiere si se logra desarrollar la actividad que se propone como proyecto de vida. Existe una dura crítica al grupo familiar de origen y un autorreproche por lo que no se hizo en las etapas formativas, lo que justifica y se vincula con la adhesión al grupo de consumo. El cambio identitario propuesto presupone dejar el consumo y la adopción de medidas de prevención a nivel sexual. La imposibilidad de "sostener" el cambio, en el caso en que se produzca, incluye a la vez el fracaso en relación con el nuevo proyecto de vida y la recaída en el consumo de drogas.

\section{Cambios en la esfera afectiva}

Se trata en este caso de cambios que tienen que ver con mantener un vínculo afectivo, básicamente con una posible pareja, pero sobre todo con los hijos. Se plantea luchar por la supervivencia pensando en los hijos, única idea que los sostiene en los momentos críticos. Se identifica el no tener ganas de vivir con la idea de que "ni siquiera el pensar en los hijos da ganas de seguir viviendo". Es interesante señalar que la presencia de los hijos en estos relatos no se refiere a contactos reales con ellos (en el caso de uno de los entrevistados, por ejemplo, hacía ocho meses que no los veía), sino a la idea de que deben vivir para "no faltar a los hijos", para que ellos sepan que tienen un padre. La vinculación entre la idea de los hijos y la abstinencia y el cumplimiento del tratamiento hace que cualquier conducta que implique apartarse de estas normas sea vivida de un modo culpógeno.

La idea de familia adquiere en estos casos un sentido identitario básico: se "es" para una familia, y si ella no está presente en el imaginario no tiene sentido vivir. Se pretende crear una versión corregida de la familia de origen, a la que se reprochan carencias básicas.

\section{La dificultad del cambio identitario}

En este caso el cambio tiene que ver con una conversión deseada pero vislumbrada como sumamente dificultosa. Si bien existe un propósito de cambio como en el tipo l, este propósito está planteado de un modo tan difuso y general, que aparece como un deseo de ser "otro" mágicamente, en la medida en que no se aclaran los pasos necesarios a seguir para ser ese "otro". La abstinencia y la continuidad del trata- 
miento se plantean como metas difíciles de alcanzar si no se cuenta con fuertes apoyos institucionales, en la medida en que se parte del supuesto de que no se puede contar con la familia de origen ni con el grupo de pares.

\section{EI no cambio}

Este tipo está representado por relatos en los que no se plantean ni cambios en el eje de la actividad, ni en el eje de los afectos. En estos relatos no aparece que los entrevistados estén preparados para intentar una nueva modalidad de gestión de su enfermedad, diferente a las discontinuidades que han protagonizado hasta ahora. Como en el tipo anterior, el vínculo familiar es débil y no se espera nada de él.

Hemos visto que la irrupción del sida, en la medida en que es caracterizado como una enfermedad que podría haber sido evitada si se hubiera modificado la conducta, conlleva en la mayoría de los casos una mirada sobre el propio pasado que, a la vez que intenta explicar el porqué se adoptó un camino que fue perjudicial para la persona, implica una autocrítica que representa, en ocasiones, una gran sobrecarga emocional.

Lo que surge de las entrevistas en su conjunto - y en esto coincidimos con lo planteado por Delor (1997) a partir de un estudio semejante realizado en Francia -, es que las personas afectadas por la enfermedad han vivido situaciones de soledad y momentos de gran tensión que los han llevado a puntos nodales en sus trayectorias vitales (en el inicio del consumo, en el momento del diagnóstico de seropositividad, frente a un límite duro como la cárcel, en el momento de la aparición de los síntomas del sida o frente a la pérdida de un ser querido).

En casi todos ellos pueden detectarse también puntos de inflexión a partir de los cuales realizaron un replanteo vital que trajo aparejados importantes cambios en la imagen de sí mismos y en sus conductas. El autocuidado y el cuidado de los demás son conductas que corren en paralelo con el replanteo a partir de los mencionados puntos de inflexión. Carricaburu y Pierret (1992) designan estos procesos como "recomposición identitaria", en la medida en que son abarcativos de muchos aspectos, incluyendo los más íntimos.

Por otra parte, no puede pensarse que la vulnerabilidad frente al riesgo del sida constituya un "rasgo" de las personas, porque esto la convertiría en un aspecto "esencial" de los sujetos. Se trata, por el contrario, de asumir la vulnerabilidad en ciertas situaciones o momentos de la trayectoria individual, y estas situaciones están marcadas por contextos sociales e interaccionales específicos. Hemos pretendido identificar, a través de los relatos de los entrevistados, algunos aspectos que los han tornado vulnerables al VIH.

Este enfoque supone, en consecuencia, un desplazamiento del eje de los individuos al eje de lo psicosocial, en tanto las situaciones que concurren a que las personas no tomen en cuenta el riesgo del sida implican otros riesgos que surgen de demandas y requerimientos emanados de la vida cotidiana, en cuya jerarquización no figura el sida. A menudo estas demandas implican riesgos de distintos tipos cuyas magnitudes resultan en una inmovilización por parte del individuo, incapaz de identificar y evaluar las amenazas. Por otra parte, como dice Delor "la reconstrucción del riesgo y del sentido atribuido a él en función de la situación social requiere de un verdadero trabajo de análisis porque se trata de retraducir lo que ha sido ya el objeto de un trabajo de traducción de parte del individuo" (1997: 22).2 Para este autor, los relatos de los entrevistados delimitan tres aspectos esenciales a tomar en cuenta en su análisis:

1. las trayectorias sociales de los individuos, que permiten identificar ciertas regularidades entre momentos específicos de vulnerabilidad;

2. las interacciones, en la medida en que ellas permiten identificar el tipo de relaciones y encuentros que han influido en la vulnerabilidad de los individuos;

3. ciertos elementos del contexto social, cuyo control escapa a las influencia directa de los individuos, y que pueden "fragilizar" sus recursos personales, debilitándolos.

La tipología presentada en relación con la gestión del riesgo frente a la enfermedad de las personas cuya vía de transmisión del VIH fue el consumo de drogas por vía endovenosa muestra que el factor que las atraviesa se refiere a los cambios adoptados (o no adoptados) a partir del diagnóstico de seropositividad en relación con el estilo de vida anterior. Esta posibilidad de cambio está fuertemente ligada a los vínculos familiares y al grupo de consumo.

\section{BIBLIOGRAFÍA}

Ariès Ph. (1960; traducción española 1990) El niño y la vida

familiar en el antiguo régimen. Madrid: Taurus.

Bertaux D. (1997) Les récits de vie. París: Nathan.

Bourdieu P. (1980) Le sens pratique. París: Éd. du Minuit.

Carricaburu D. y Pierret J. (1992) Vie qutotidienne et recompositions identitaires autour de la séropositivité. París: CERMES-ANRS (Mimeo).

2 Bertaux (1997) califica a esta tarea como hermenéutica, en la medida en que implica el desciframiento del sentido a partir de los relatos. 
Cohen Ph. (1972) "Subcultural conflict and working class community. Working Papers in Cultural Studies". University of Birmingham, 2: 5-51.

Delor F. (1997) Séropositifs. Trajectoires identitaires et rencontres de risque. París: L'Harmattan.

De Singly F. (1993, 2a.edición) Sociologie de la famille contemporaine. París: Nathan.

Donzelot J. (1977) La police des familles. Paris: Éd. de Minuit.

Durkheim E. (1888) Introduction a la sociologie de la famille. Annales. Bordeaux: Faculté des Lettres.

Feixa C. (1999, 2a. edición) De jóvenes, bandas y tribus. Barcelona: Ariel

Foucault M. (1990) Vigilar y castigar. Madrid: Siglo XXI.

Giddens A. (1991) La transformación de la intimidad. Madrid: Ed. Cátedra.

Giddens A. (1999) La tercera vía. Madrid: Taurus.

Glaser B. y Strauss A. (1967) The discovery of grounded theory. Strategies for qualitative research. Chicago: Aldine.

Hall S. y Jefferson T. (Edits.) (1983) Resistance through rituals. Youth subcultures in post-war Britain. Londres: Hutchinson.
Hareven T. (1991) "The history of the family and the complexity of social change". The American Historical Review 96, 1: 95-124.

Kornblit A. L., Petracci M. y Mendes Diz A. M. (1999) "Ser hombre, ser padre". En AEPA/CEDES/CENEP: Avances en la investigación social en salud reproductiva $y$ sexualidad. Trabajos presentados en el Tercer Taller de Investigaciones Sociales en Salud Reproductiva y Sexualidad. Buenos Aires, 12-14 de agosto de 1998.

Laclau E. (1991) New reflections on the revolution of our time. Londres: Verso.

Langellier K. y Peterson E. (1997) “Las historias de la familia como estrategia de control social". En D. Mumby (comp.) Narrativa y control social. Buenos Aires: Amorrortu.

Lyotard J-F. (1985) The postmodern condition. Manchester: Manchester University Press.

Moscovici S. (1996, 2a. edición) Psicología de las minorías activas. Madrid: Morata

Sautu R. (1999) El enfoque biográfico. Buenos Aires: Editorial de Belgrano.

Segalen M. (1996, 4a. edición) Sociologie de la famille. París: A. Colin. 
